\title{
An overview on germinated brown rice and its nutrigenomic implications
}

\begin{abstract}
White rice is a major staple food for people in low to middle income countries and it can increase the risk of cardiometabolic disease. Brown rice, especially when germinated, is a healthier alternative. Various functional properties have been documented for the bioactiverich germinated brown rice. Nutrigenomic studies, dwelling on interactions at diet-genome interface, have expanded our understanding of the role of diets on health. The nutrigenomic basis for the functional properties of GBR have also been reported; its antihyperglycemic, hypo cholesterolemic, and antioxidative effects are mediated by its bioactives, partly via transcriptional regulation of genes involved in gluconeogenesis, cholesterol metabolism, and oxidative stress, respectively. Additionally, GBR's ability to improve menopausal symptoms is reported to be due to its ability to upregulate bone metabolism and uterine estrogen related genes, and downregulate inflammatory genes. Food synergy plays a role in the overall functional effects of GBR. Further studies on proteomics, metabolomics, nutria kinetics, and nutria dynamics are indicated.
\end{abstract}

